\title{
Induction of the unfolded protein response by cigarette smoke is primarily an activating transcription factor 4-C/EBP homologous protein mediated process
}

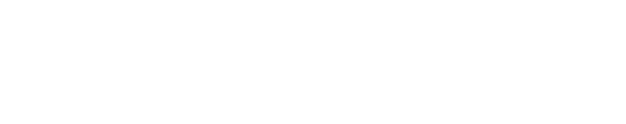

Patrick Geraghty

Alison Wallace

Jeanine M D'Armiento

Department of Medicine, Divisions of Molecular and Pulmonary Medicine, Columbia University College of Physicians and Surgeons, New York, NY, USA
Correspondence: Jeanine D'Armiento Department of Medicine, Divisions of Molecular and Pulmonary Medicine, Institute of Human Nutrition, Columbia University College of Physicians and Surgeons, New York, NY 10032, USA $\mathrm{Tel}+\mathrm{I} 2$ I 23053745

Fax + I 212305 I I88

Email jmdI2@columbia.edu
Purpose: Cigarette smoke is the major risk factor associated with the development of chronic obstructive pulmonary disease (COPD). Recent studies propose a link between endoplasmic reticulum (ER) stress and emphysema, demonstrated by increased ER stress markers under smoking conditions. Here, we investigate whether cigarette smoke-induced ER stress is cell specific and correlates with acute and chronic cigarette smoke exposure.

Methods: Gene and protein expression changes in human primary lung cell cultures following cigarette smoke extract (CSE) exposure were monitored by qPCR and Western blot analysis. Mice and guinea pigs were exposed to cigarette smoke and ER stress markers examined in whole lung homogenates. Inflammatory cells from the bronchoalveolar lavage fluid of 10 days smoke exposed mice were also examined.

Results: Cigarette smoke induced a trend increase in the ER stress response through an activating transcription factor 4 (ATF4) mediated induction of $\mathrm{C} / \mathrm{EBP}$ homologous protein (CHOP) in primary small airway epithelial cells. Bronchial epithelial cells and macrophages responded similarly to CSE. Wild-type mice and guinea pigs exposed to acute levels of cigarette smoke exhibited increased levels of CHOP but not at significant levels. However, after long-term chronic cigarette smoke exposure, $\mathrm{CHOP}$ expression was reduced. Interestingly, inflammatory cells from smoke exposed mice had a significant increase in CHOP/ATF4 expression.

Conclusion: A trend increase in CHOP levels appear in multiple human lung cell types following acute cigarette smoke exposure in vitro. In vivo, inflammatory cells, predominately macrophages, demonstrate significant cigarette smoke-induced ER stress. Early induction of CHOP in cigarette smoke may play a pivotal role in early induction of lung disease, however in vivo long-term cigarette smoke exposure exhibited a reduction in the ER stress response.

Keywords: COPD, ER stress, cigarette smoke, CHOP

\section{Introduction}

Cigarette smoke is the leading risk factor associated with the development of chronic obstructive pulmonary disease (COPD). ${ }^{1}$ COPD is the third largest cause of death in the $\mathrm{USA}^{2}$ and fourth worldwide, ${ }^{1}$ and is characterized by progressive, irreversible airflow limitation caused by emphysema, airway remodeling, and chronic bronchitis. ${ }^{3}$ Increased inflammation, oxidative stress, impaired cell repair, protease/antiprotease imbalance, cell death, and destruction of the extracellular matrix are all observed in COPD lungs and are all believed to contribute to disease progression. Over the past 3 years, several investigators have demonstrated an ER stress response to cigarette smoke and suggested 
that induced endoplasmic reticulum (ER) stress may be an additional factor for COPD progression. ${ }^{4-10}$

Under normal conditions, the ER is involved in protein biosynthesis, correct folding, and posttranslational modifications of secretory and membrane proteins. Stress to the ER results in the unfolded protein response (UPR), whereby chaperones clear unfolded proteins to elevate cellular stress. If ER stress is persistent, it can lead to apoptosis via altered expression of cytosine-cytosine-adenine-adenine-thymine enhancer-binding protein homologous protein (CHOP). ${ }^{11}$ The localization of resident ER proteins changes following stress, thereby serving as markers for the ER stress response. Under normal conditions, binding immunoglobulin protein (BiP) also known as $78 \mathrm{kDa}$ glucose-regulated protein (GRP-78) physically interacts with the luminal domain of membrane-bound proteins, such as inositol-requiring enzyme 1 (IRE1) and protein kinase RNA-like endoplasmic reticulum kinase (PERK). ${ }^{12}$ Under stress conditions, BiP dissociates from the membrane-bound proteins and associates permanently with misfolded, under-glycosylated or incompletely assembled proteins. ${ }^{13,14}$ Since cigarette smoke exposure leads to increased ER stress markers in the lungs of patients with COPD,${ }^{8}$ it is speculated that ER stress contributes to the pathogenesis of COPD. Interestingly, urban particulate matter has also been shown to cause ER stress and the unfolded protein response in human lung cells. ${ }^{15}$ However, studies have not examined the ER stress response in multiple primary nontransformed human lung cell types and in animal smoke exposure models other than mice.

In this study we investigated the effect of cigarette smoke on multiple human primary lung cell types with respect to the initiation of the ER stress response pathway. To evaluate this hypothesis, epithelial, endothelial, and macrophages cells were examined following exposure to cigarette smoke extract; lung inflammatory cells from bronchoalveolar lavage (BAL) fluid of smoke exposed mice were also analyzed. Additionally, mice and guinea pigs were exposed to cigarette smoke and subsequent changes in CHOP expression were examined.

\section{Material and methods Primary human cell culture}

Human small airway epithelial (SAE) cells, human bronchial epithelial (HBE) cells, and human pulmonary endothelial (HMVEC) cells were cultured according to the manufacturer's instructions (Clonetics, San Diego, CA). THP-1 monocytes were cultured in RPMI 1640 medium (Invitrogen, Carlsbad, CA) and were differentiated to macrophage-like cells with 48 hours of exposure to phorbol 12-myristate 13-acetat (PMA). The macrophage-like cells were incubated with fresh medium daily for a further 5 days before stimulation. Cells between passages 2 and 6 were used in all in vitro experiments.

\section{Reagents used}

Unless specified, all reagents were purchased from Sigma Aldrich (St Louis, MO). Tunicamycin and thapsigargin stocks were prepared in dimethyl sulfoxide (DMSO) and diluted $\mathrm{E} 10^{5}$ in culture media prior to addition to cells. Treatment conditions used were $1 \mu \mathrm{M}$ thapsigargin and $1 \mu \mathrm{M}$ tunicamycin.

\section{Preparation of cigarette smoke extract and cell treatment}

Cigarette smoke extract (CSE) was prepared using a modified protocol. ${ }^{16}$ Briefly, a Barnant vacuum pump operating at constant airflow was used to draw the smoke of one 3R4F research grade cigarette (University of Kentucky) through $25 \mathrm{~mL}$ of Dulbecco's phosphate-buffered saline. This solution $(100 \% \mathrm{CSE})$ was adjusted to $\mathrm{pH} 7.4$, treated with an endotoxin removal reagent as described by manufacturers (20484; MoBio, Carlsbad, CA), sterile filtered $(0.22 \mu \mathrm{m})$ and added to growth medium to a final concentration of $5 \%$ or $10 \%$ within 60 minutes of preparation. Cell viability was accessed following CSE exposure using the alamarBlue kit (Invitrogen) according to the manufacturer's specifications.

\section{Animals}

To determine the in vivo effects of smoke exposure, 8-weekold C57BL/CBA mice were exposed to cigarette smoke in a specially designed smoking chamber (Teague Enterprises, Davis, CA) and this method was validated as previously described. ${ }^{17-19}$ Briefly, mice ( $n=5$ for each time point) were exposed daily to 6 hours of mainstream (active) and sidestream (passive) smoke from $2 \mathrm{R} 4 \mathrm{~F}$ research grade cigarettes (University of Kentucky, Louisville, KY) for 5 days/week for 4 weeks or 1 year. Control mice were exposed to room air ( $\mathrm{n}=5$ for each time point). Animals were provided food and water ad libitum. Mice were sacrificed by an overdose of isoflurane. The lung was homogenized in RIPA protein lysis buffer (Cell Signaling, Danvers, MA) and $100 \mu \mathrm{g}$ of protein was analyzed by immunoblotting. Additionally, C57BL/CBA mice exposed to 10 days of cigarette smoke were sacrificed and BAL fluid collected for RNA analysis on inflammatory cells gene expression changes. Hartley strain guinea pigs (Wilmington, MA) were also exposed to cigarette 
smoke for 12 weeks, under the same conditions described above $(\mathrm{n}=10)$, which developed emphysema as previously described. ${ }^{19}$ Following smoke exposure, lungs were collected for protein analysis. The Animal Care and Use Committee of Columbia University approved all the experiments.

\section{qPCR analysis}

Total RNA was isolated from cells or mouse lung tissue using the Qiagen RNeasy Mini Kit (Qiagen Inc, Valencia, CA) as described by the manufacturer. Gene transcript levels of CHOP, XBP1, ATF-4, ATF-6, and two housekeeping genes (ACTB and GAPDH) were quantified by real-time PCR with the use of an ABI Prism ${ }^{\circledR} 7900$ HT Sequence Detection System (Applera Corporation, Norwalk, CT). TaqMan ${ }^{\circledR}$ Gene Expression Assays were purchased from Applied Biosystems (Foster City, CA).

\section{X-box binding protein I (XBPI) splicing analysis}

RT-PCR of XBP1 mRNA was performed essentially as described previously. ${ }^{20}$ In brief, $2 \mu$ g of total RNA was reversetranscribed with High-Capacity cDNA Reverse Transcription Kit (Applied Biosystems) and amplified with Promega PCR master-mix (Madison, WI) using the following pair of primers: 5'-AAACAGAGTAGCAGCTCAGACTGC-3' and 834-853 5'-TCCTTCTGGGTAGACCTCTGGGAG-3' for XBP1. Amplified fragments covering a 26-nt intron (with a Pst1 site) and flanking exon fragments were digested with Pst1 and separated on 3\% agarose gels. cDNA was visualized by staining with ethidium bromide and detected by an image analyzer (model FLA-3000; Fuji, Tokyo, Japan).

\section{Immunoblotting}

Cell monolayers were removed by scrapping in cold phosphate-buffered saline and resuspended in $100 \mu \mathrm{L}$ of protein lysis buffer (50 mM HEPES, pH 7.5, $150 \mathrm{mM} \mathrm{NaCl,} \mathrm{1 \%}$ Triton X-100, 1\% glycerol, $1 \mathrm{mM}$ EDTA, $10 \mathrm{mM} \mathrm{NaF}$, $2 \mu \mathrm{g} / \mathrm{mL}$ leupeptin, $1 \mu \mathrm{g} / \mathrm{mL}$ pepstatin $\mathrm{A}, 10 \mathrm{mM} \mathrm{Na}_{3} \mathrm{VO}_{4}$, and $1 \mathrm{mM}$ phenylmethylsulfonyl fluoride), and $20 \mu \mathrm{g}$ of protein was separated on $12 \%$ SDS-polyacrylamide gels and transferred to nitrocellulose membranes. Rabbit antibodies against GRP78 (Cell signaling; \#3183), Phospho-PERK (Thr980) (16F8) (Cell Signaling; \#3179), CHOP (Santa Cruz Biotechnology, Santa Cruz, CA, USA; sc-793) and Actin (Santa Cruz; sc-1616) were detected with enhanced chemiluminescence reagents (Pierce, Thermo Scientific, Rockford, IL).

\section{Immunohistochemistry}

Paraffin-embedded samples were sectioned at $6 \mu \mathrm{m}$. Nonenzymatic unmasking was performed in a Tris-EDTA buffer (10 mM Tris Base, $1 \mathrm{mM}$ EDTA, 0.05\% Tween 20, $\mathrm{pH}$ 9.0). Endogenous peroxidase was quenched by 10-minute incubation in $3 \% \mathrm{H}_{2} \mathrm{O}_{2}$. Sections were stained with polyclonal anti-CHOP (sc-793; Santa Cruz Biotechnology, Santa Cruz, CA) and anti-GRP78/BiP (Cell signaling; \#3183) antibodies, both raised in rabbits. Detection was by an avidin-biotin complex (ABC)-based method (Vector Laboratories, Burlingame, CA) using a biotinylated secondary antibody, which is linked to an ABC-enzyme complex, alkaline phosphatase or horseradish peroxidase. Alkaline phosphatase complexes were detected with permanent liquid red (Dako, Glostrup, Denmark) yielding a red reaction product or 3,3' Diaminibenzidine (Vector Laboratories) was used as a substrate for peroxidase complexes yielding a brown reaction product at the site of the target antigen. Sections were counterstained with hematoxylin (blue).

\section{Statistical analysis}

Results are expressed as mean \pm SEM. Statistics were performed by analysis of variance and Student's $t$ test. All tests were performed using Prism GraphPad (Graphpad Software, Inc, San Diego, CA). A $P$-value of less than 0.05 was considered to indicate statistical significance.

\section{Results \\ CHOP expression following cigarette smoke extract exposure in small airway epithelial cells, bronchial epithelial cells, and macrophages}

When cells are undergoing ER stress, misfolded protein accumulation leads to GRP78 dissociation from the luminal domain of the membrane-bound proteins, IRE1 and PERK, ${ }^{12}$ resulting in autophosphorylation and activation of these proteins, ultimately leading to $\mathrm{CHOP}$ expression. ${ }^{11}$ Several recent papers have demonstrated that cigarette smoke can induce GRP78 and CHOP in A549, HBE, and mouse fibroblast cells (ATCC CCL-92). ${ }^{469,10}$ SAE cells were primarily used throughout the present study as the small airways play a vitally important role in COPD progression. ${ }^{3,21,22}$ CSE was processed to remove endotoxin prior to treating cells in order to eliminate endotoxin induced inflammation. ${ }^{23} \mathrm{SAE}$ cells were stimulated with various concentrations of CSE. Previously, high levels of CSE had been applied to induce an ER stress response. ${ }^{9}$ High levels of CSE result in reduced SAE cell viability (Figure 1A) without an induction of CHOP (Figure 1B). 
A

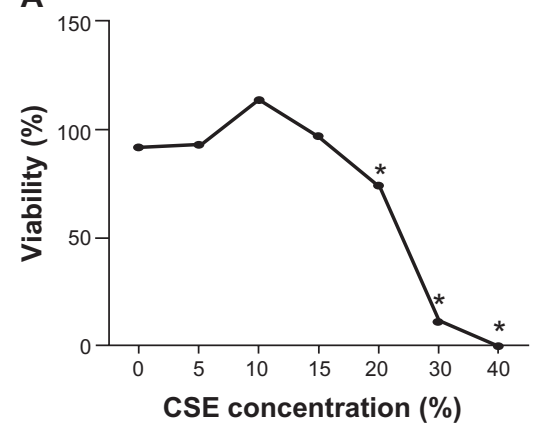

B

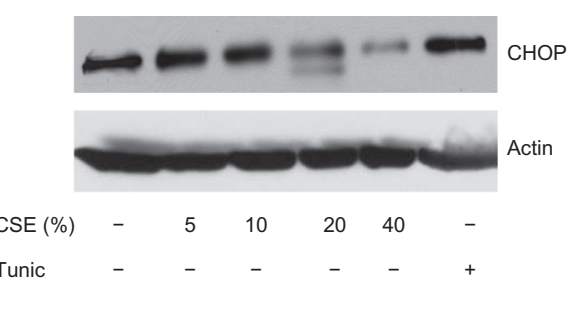

C

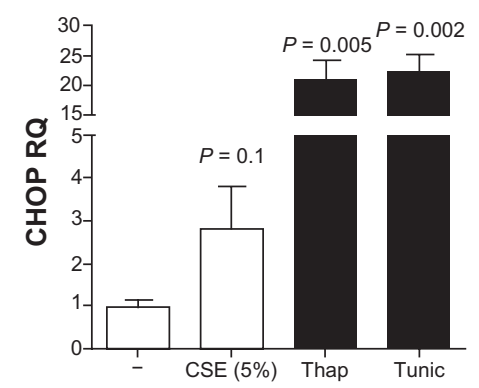

D

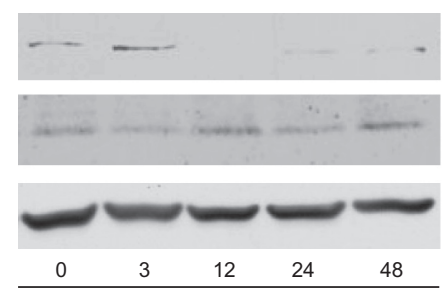

5\% CSE

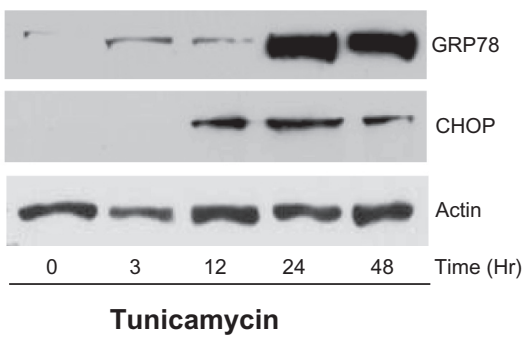

E

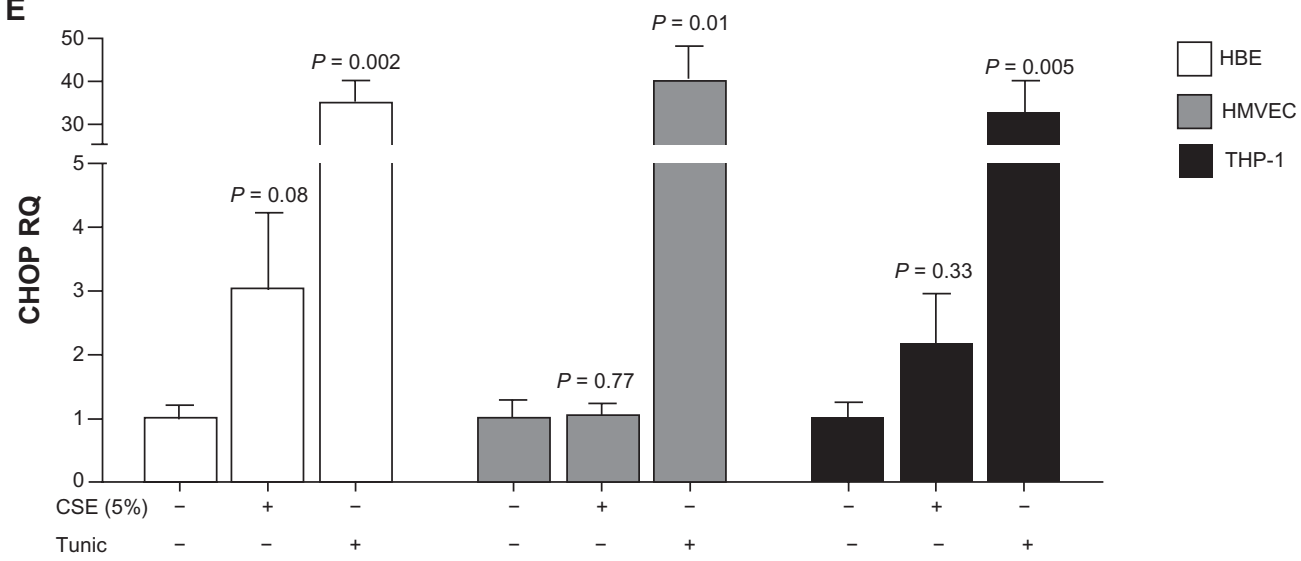

$\mathbf{F}$

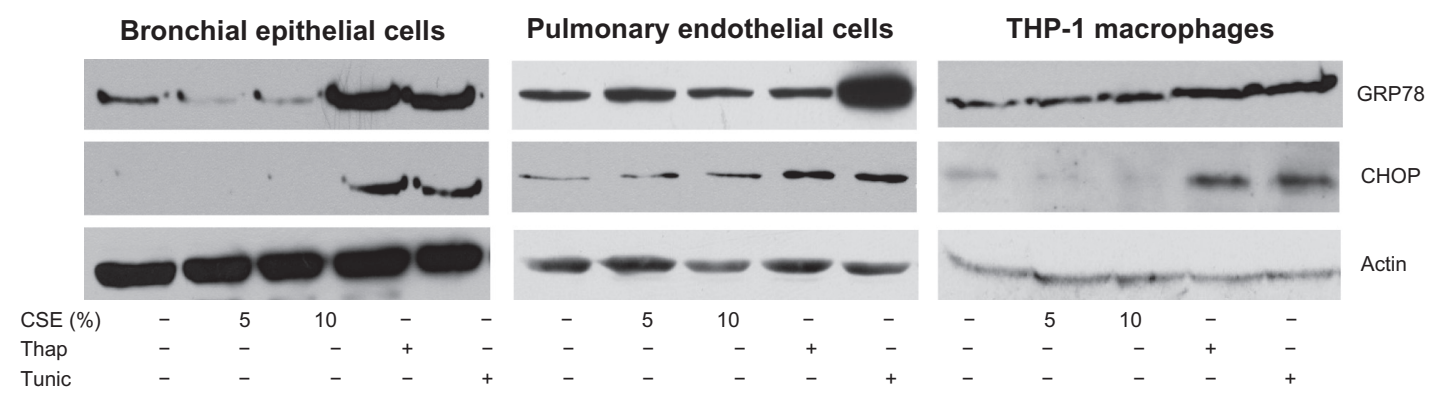

Figure I Cigarette smoke extract induces a modest induction of CHOP in SAE cells, bronchial epithelial cells, and macrophages. SAE cells were exposed to various concentrations of CSE for 24 hours and high CSE concentrations result in reduced viability (A) and no increase of CHOP protein levels (B). C) CHOP protein levels were elevated following 24 hours exposure to $5 \%$ CSE, demonstrated by qPCR. Exposures to thapsigargin (I $\mu \mathrm{M})$ or tunicamycin (I $\mu \mathrm{M})$ were used as positive ER stress controls. D) This CSE induction of CHOP protein was undetectable from I to 48 hours, by Western blot. A modest induction of CHOP is observed in HBE and THP-I cells but not in HMVEC following 5\% CSE exposure for 24 hours, at the mRNA level (E) but undetectable by Western blot analysis (F).

Notes: Each assay was performed on 4 independent days and graphs are represented as mean \pm S.E.M. P-values shown, comparing cells not treated to CSE.

Abbreviations: CHOP, C/EBP homologous protein; CSE, cigarette smoke extract; ER, endoplasmic reticulum; HBE, human bronchial epithelial; HMVEC, human pulmonary endothelial cells; SAE, small airway epithelial. 
A trend towards an increase in CHOP mRNA expression is observed following 5\% CSE exposure to SAE cells but the change from baseline does not reach statistical significance $(P=0.1)$ (Figure 1C). A study by Jorgensen and colleagues suggested that prolonged exposure of CSE is needed to observe an increase in CSE-induced GRP78. ${ }^{5}$ Prolonged exposure to tunicamycin and thapsigargin exhibited increased CHOP from 12 hours of stimulation and GRP78 protein expression observed at 24 hours (Figure 1D). However, despite the trend towards an increase in ER stress markers at the mRNA level following CSE exposure there was no significant difference of $\mathrm{CHOP}$ and GRP78 observed at the protein level (Figure 1D).

Following only a minimal change in CHOP mRNA expression in SAE cells, other pulmonary cells (HBE, HMVEC, and THP-1 macrophages) were stimulated with CSE to investigate changes in CHOP and GRP78. The data suggested a statistically insignificant trend towards an increase in CSE induced CHOP gene expression in $\mathrm{HBE}$ cells $(P=0.08)$ (Figure 1E). Interestingly, macrophages $(P=0.33)$ and $\operatorname{HMVEC}(P=0.77)$ had no significant change of CHOP expression from baseline after CSE stimulation. Similar to SAE cells, the other cell types exhibited unaltered levels of GRP78 and CHOP following CSE exposure, at the protein level (Figure 1F). Therefore, although it is possible that an ER stress response may occur at very low levels in SAE cells, HBE cells, and macrophages, we do not observe a significant change in $\mathrm{CHOP}$ expression at multiple time points and CSE concentrations.

\section{CSE altered CHOP expression is primarily due to phosphorylation of PERK branch of CHOP expression in SAE cells}

The three branches of inducible CHOP expression were then examined. Cigarette smoke treatment did not significantly increase the levels of ATF6 (Figure 2A) or XBP1 (Figure 2B) following CSE stimulation in SAE cells. Splicing of XBP1 mRNA is required for XBP1 protein expression. ${ }^{20,24}$ Upon ER stress, the conformational alteration of IRE1 via phosphorylation exposes a ribonuclease capability that removes an intron from XBP1 mRNA, resulting in the generation of a functional protein that is a potent transcriptional regulator of genes involved in protein folding and degradation, two necessary mechanisms needed to restore ER homeostasis. SAE cells were exposed to CSE over multiple time points and RNA samples were collected for XBP1 splicing analysis. CSE exposure did not increase XBP1 mRNA splicing (Figure 2C; 3 hour CSE exposure depicted in this figure). Another mediator protein, PERK, was examined for phosphorylation. GRP78 dissociation activates PERK, which in turn phosphorylates eIF2alpha, an inhibitor of new protein translation and activator of the transcription factor ATF4. Following CSE exposure, phosphorylation of PERK was observed in primary SAE cells (Figure 2D) and a significant increase in the expression of ATF4 was observed $(P=0.004)$ (Figure $2 \mathrm{E})$. Therefore this data indicates that the predominant signaling of CHOP expression in $\mathrm{SAE}$ cells appears to be the PERK-ATF4 branch.

\section{$\mathrm{CHOP}$ expression altered in mouse lungs following cigarette smoke exposure}

Multiple cell types and various stress conditions can selectively activate one or more of the ER sensors. ${ }^{25}$ With this in mind, an in vivo approach was undertaken to investigate the possible induction of ER stress in C57BL/CBA mice exposed to cigarette smoke. The mice were exposed to cigarette smoke to induce acute inflammatory response (4 weeks) and a chronic inflammatory response (1 year). As described previously, ${ }^{17}$ following 4 weeks of cigarette smoke exposure, mice exhibit minimal lung destruction but 1 year of exposure results in a statistically significant increase in tissue destruction. Interestingly, CHOP mRNA levels display a trend towards a slight increase following 1 month of cigarette smoke exposure compared to control levels, which did not reach significance levels (Figure 3A). In contrast, 1 year of smoke exposure resulted in a significant reduction in CHOP expression $(P=0.015)$ (Figure 3A) with a trend reduction in ATF4 expression (Figure $3 \mathrm{~B} ; P=0.1$ ). ATF4 is significantly decreased in 1 year smoke exposed mice compared to 1 month cigarette smoke exposed mice $(P=0.041)$. The protein levels of GRP78 are unaltered in acute (Figure $3 \mathrm{C}$ ) and chronically cigarette smoke exposed mice (Figure 3D). CHOP protein levels are unaltered in acute smoke exposed mice (Figure $3 \mathrm{C}$ ) as seen in the cell culture models but reduced in the mice exposed chronically to smoke (Figure 3D) compared to their corresponding nonexposed litter mates. Phosphorylation of PERK in the mice was similar to the $\mathrm{CHOP}$ protein profiles (Figures $3 \mathrm{C}$ and D). Immunohistochemical examination of GRP78 and CHOP in mouse lung tissue exposed for 1 year to smoke demonstrated that both ER stress markers are present primarily in bronchial epithelial cells (Figure 3E) but surprisingly the smoke exposed lungs appeared to have a similar CHOP and GRP78 cell profile when compared to room air exposed lungs (Figure 3E).

\section{Cigarette smoke exposure and $\mathrm{CHOP}$ in guinea pig lungs}

Following our observations of a possible trend towards an increase in $\mathrm{CHOP}$ expression in mouse lungs following 
A

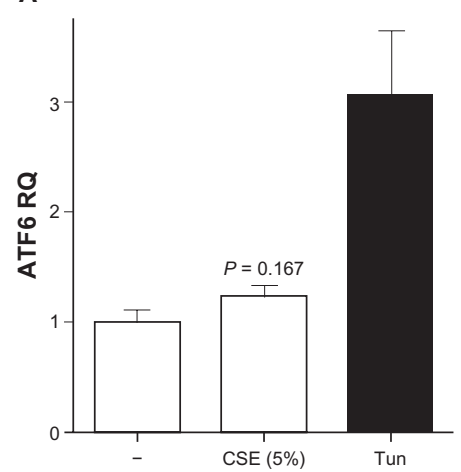

B

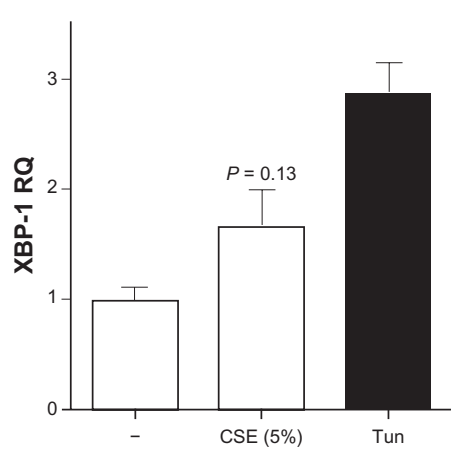

C

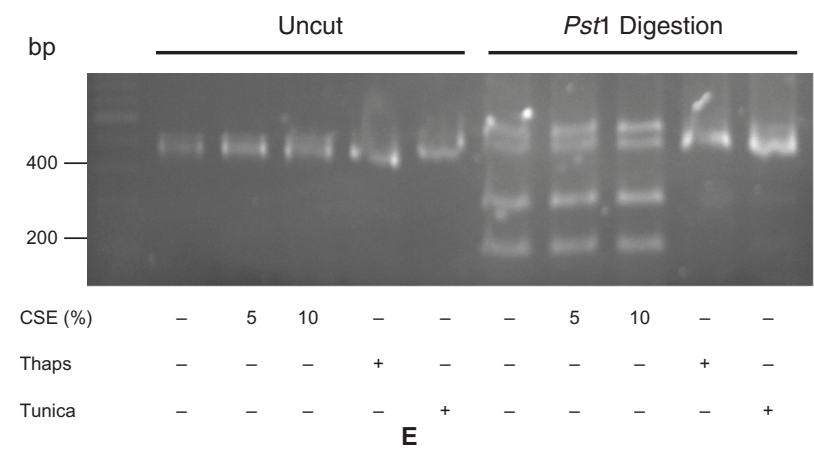

D
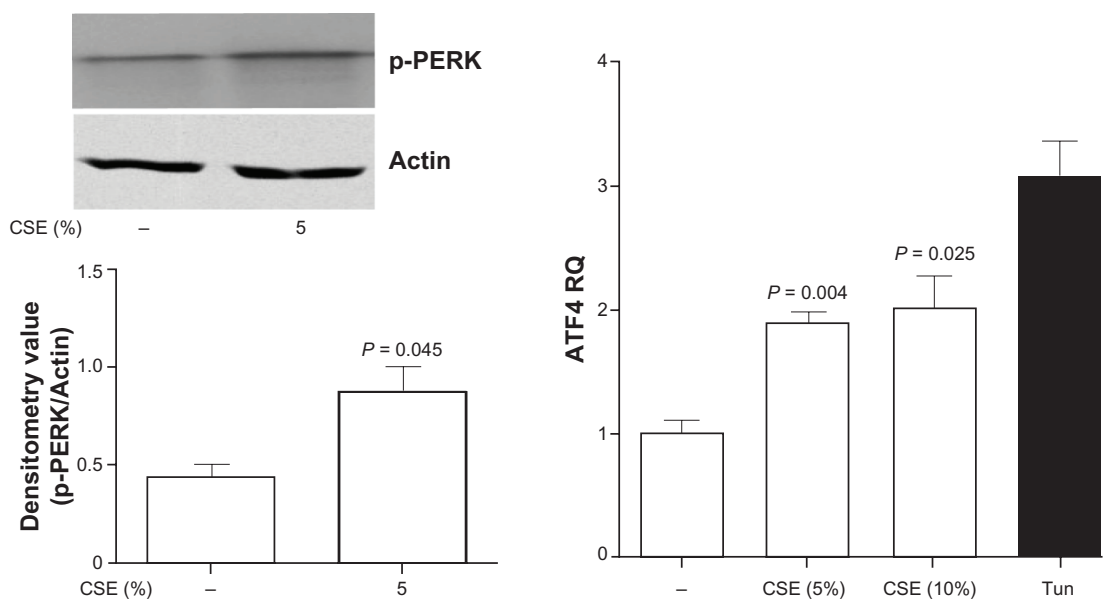

Figure 2 Cigarette smoke extract exposure appears to induce CHOP expression in an ATF4 signaling cascades in SAE cells. A) AFT6 and B) XBP-I levels were examined in SAE cells by qPCR, following 5\% CSE exposure for 24 hours. C) XBPI PCR samples were examined on $3 \%$ agarose gel uncut or following Pst I digestion. SAE cells were exposed to CSE (5 or 10\%), thapsigargin or tunicamycin for 3 hours. XBPI splicing was examined on uncut and Pst I digested XBPI amplified cDNA. D) Phosphorylation of PERK was examined by Western blot following small airway epithelial cells exposed to $5 \%$ cigarette smoke extract for I hour. Densitometry confirmed an increase in phosphorylation of PERK. E) ATF4 levels were examined in SAE cells by qPCR, following $5 \%$ and $10 \%$ CSE exposure for 24 hours.

Notes: Each assay was performed on 4 independent days and $P$-values shown, comparing CSE exposed cells to control.

Abbreviations: CHOP, C/EBP homologous protein; CSE, cigarette smoke extract; SAE, small airway epithelial.

cigarette smoke, we determined the influence of cigarette smoke on CHOP expression in an additional animal model. Guinea pigs were exposed to smoke for 12 weeks as previously described. ${ }^{19} \mathrm{We}$, and others, have demonstrated that the guinea pig smoking model represents an alternative means to mice for examining cigarette smoke-induced emphysema. ${ }^{19}$ Guinea pigs were exposed to cigarette smoke for 12 weeks and CHOP levels were examined by Western blot analysis. Twelve weeks of smoke exposure led to a borderline significant increase in CHOP levels $(P=0.08)$, observed by Western blot (Figures 4A and B).

\section{Cigarette smoke induces $\mathrm{CHOP}$ and ATF4 expression in alveolar inflammatory cells in wild-type mice}

Due to the limitations in understanding CHOP and ATF4 expression profiles from whole lung homogenates, we examined inflammatory cells, which were predominately 


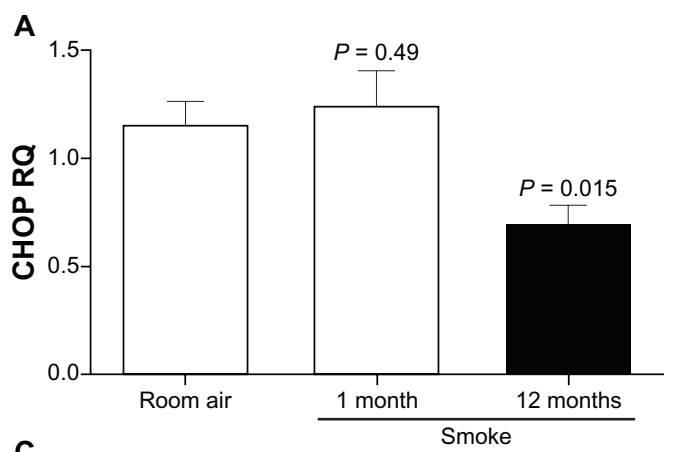

C

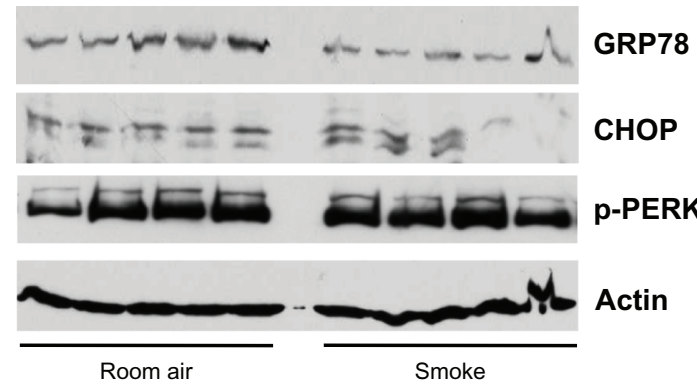

E
B

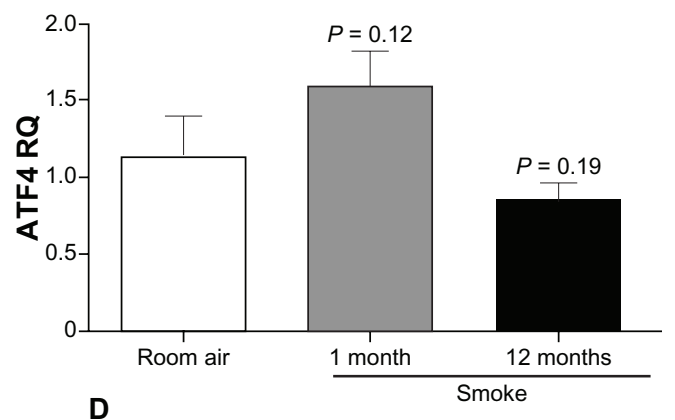

D

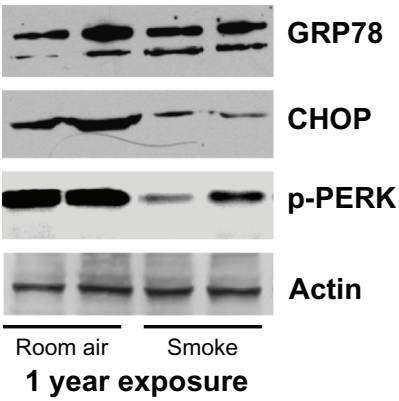

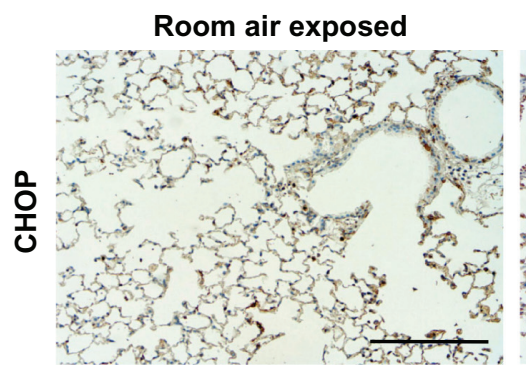
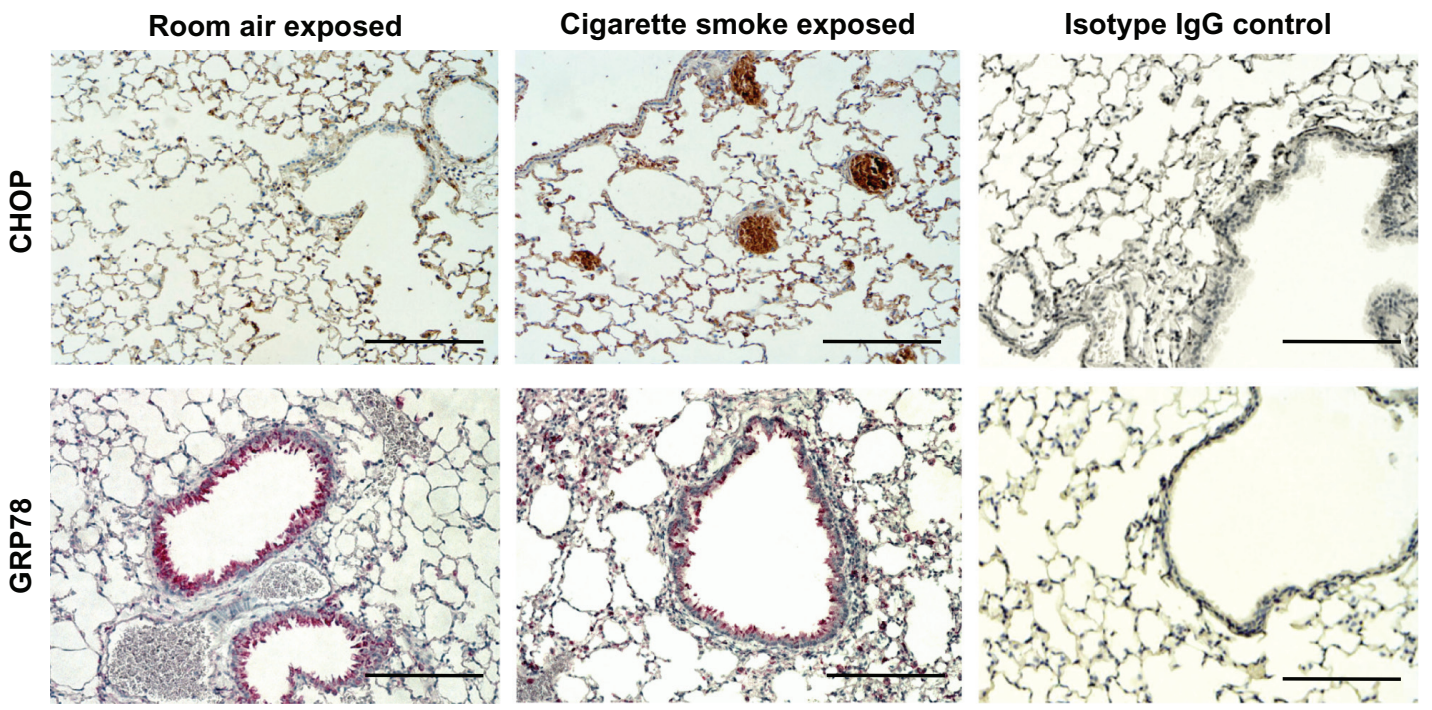

Figure 3 Acute cigarette smoke exposure does not significantly increase CHOP expression in mouse lungs, which is lost over time with chronic exposure. C57BL/CBA wild-type mice were exposed to one month or one year of cigarette smoke ( $n=5$ for each group). (A) qPCR was performed to quantify CHOP levels in each mouse group. $P$ values shown, comparing mice not exposed to cigarette smoke. (B) AFT4 levels were increased in I month cigarette smoke exposed mice. $P$-values shown, comparing to mice exposed to room air. When comparing I year to I month cigarette smoke exposed mice $P=0.041$. Western blot for GRP78, CHOP and actin were performed on lung homogenates from mice exposed to smoke for either I month (C) or I year (D). (E) One year of cigarette smoke exposure mouse lungs (middle panels) and control mice (left panels) were examined for CHOP (brown stain) and GRP78 (red stain) staining by immunohistochemistry. Scale bars represent $100 \mathrm{~mm}$. Isotype lgG represents negative control (right panels).

Abbreviation: $\mathrm{CHOP}, \mathrm{C} / \mathrm{EBP}$ homologous protein.

alveolar macrophages, for expression of CHOP and ATF4 following exposure to cigarette smoke for 10 days. We observed a significant increase in inflammatory cells recovered following exposure to cigarette smoke (Figure 5A). Ten days of smoke exposure lead to a significant increase in CHOP levels (Figure 5B; $P=0.011$ ) and ATF4 (Figure 5C; $P=0.004)$ gene expression, as observed by qPCR.

\section{Discussion}

The principle signaling mechanism of COPD is yet to be fully elucidated. Oxidative stress, inflammation, impaired cell repair, cell death, and destruction of extracellular matrix all contribute to COPD pathogenesis. In the present study, we find a significant increase in CHOP and ATF4 expression in mouse lung inflammatory cells following 10 days of cigarette smoke 

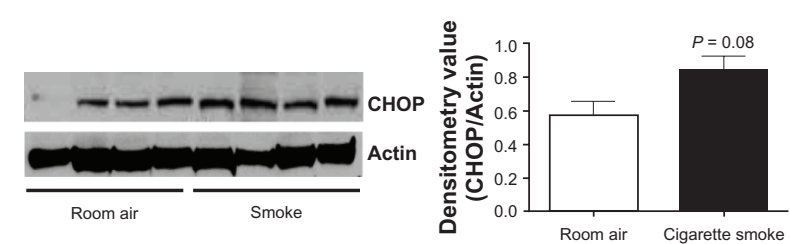

Figure 4 Cigarette smoke exposure induces a trend increase in $\mathrm{CHOP}$ expression in guinea pig lungs. Guinea pigs ( $n=4$ for each group) exposed to 12 weeks of cigarette smoke were sacrificed, protein collected from their lungs and $\mathrm{CHOP}$ and actin Western blots were performed (A). B) Densitometry confirmed a trend increase in CHOP protein levels.

Note: $P$-values shown, comparing guinea pigs exposed to room air.

Abbreviation: CHOP, C/EBP homologous protein.

exposure. However, we have not observed a significant alteration in ER stress signaling following cigarette smoke exposure in whole lung homogenates of wild-type mice or human cells, but there is a trend that cigarette smoke exposure induces the PERK/ATF4 branch of CHOP expression, as observed in guinea pigs exposed to cigarette smoke. Our results are somewhat compatible with studies that demonstrate ER stress following cigarette smoke exposure but the lack of ER stress in long-term smoke exposure and the low level of CHOP induction suggests that cigarette smoke-induced CHOP induction may not play a major role in disease progression.

This study is the first to investigate the effect of cigarette smoke on ER stress in guinea pig lungs and mouse lung inflammatory cells. Previous studies examining cigarette smoke-induced ER stress demonstrated that CSE or cigarette smoke condensate induce an ER stress response in 3T3-L1 preadipocytes, ${ }^{4}$ A549 cells, ${ }^{5}$ primary human bronchial epithelial cells, ${ }^{5}$ SA $3 \mathrm{~T} 3$ mouse fibroblasts, ${ }^{10}$ 16-HBE, ${ }^{9}$ human bronchial epithelial cells (Beas2B), ${ }^{6-8}$ and HEK$293^{7}$ cells. The range of concentrations of CSE reported to induce ER stress varies from 4\%-40\%. . $^{-10}$ The ER stress markers reported to be induced by CSE in prior studies are
GRP78, PERK, eIF2 $\alpha$, ATF4, calrecticulin, protein disulfide isomerase, and $\mathrm{CHOP}^{4-10}$ In our study there was a trend towards an increase in the PERK/ATF4 induced CHOP in primary human SAE cells exposed to 5\%-10\% CSE, but not to the extent seen in other cell types. ${ }^{4-10}$

Cigarette smoke-induced ER stress has been observed in mice ${ }^{6,7}$ and human $\mathrm{COPD}^{7,8}$ but we observed a trend increase in ER stress markers at acute cigarette smoke exposure in animals. Interestingly, human lung samples from smokers have increased UPR markers, compared to nonsmokers and ex-smokers. ${ }^{9}$ C57BL/6J mice exposed to cigarette smoke for 9 weeks induced ER stress markers, GRP78 and CHOP, in the lung. ${ }^{6} \mathrm{We}$ observe a minor trend for $\mathrm{CHOP}$ induction in C57BL/CBA mice exposed to 4 weeks of cigarette smoke but reduced CHOP expression following chronic exposure to cigarette smoke, when examining whole lung homogenates. The reduction in CHOP expression in chronic cigarette smoke exposed mice is not obvious when investigated by IHC, however, qPCR and Western blot analysis demonstrated reduced CHOP expression. Interestingly, significant ER stress is observed when examining cells collected from BAL fluid of mice exposed to cigarette smoke. Our experimental approach highlights the limitation of current approaches employed to investigate ER stress in the lung and the importance of analyzing human disease tissue microscopically to completely assess cell specific gene expression, compared to whole tissue homogenates.

Other studies also suggest that the UPR response exists to minimize the stress of oxidative protein folding. ${ }^{26}$ Furthermore, one study speculated that upregulation of UPR regulatory proteins by cigarette smoke may provide a pro-survival phenotype and may play a role in cancer cell survival. ${ }^{5}$ Cigarette smoke induction of ER stress is also associated
A

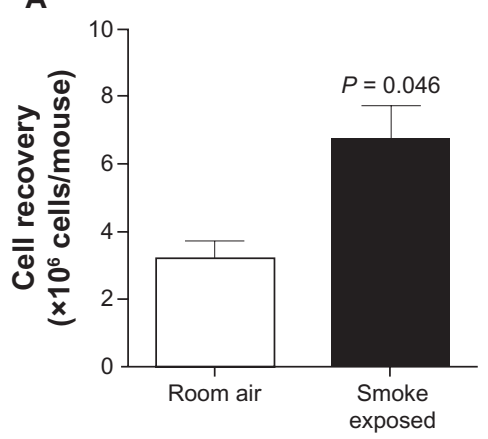

B

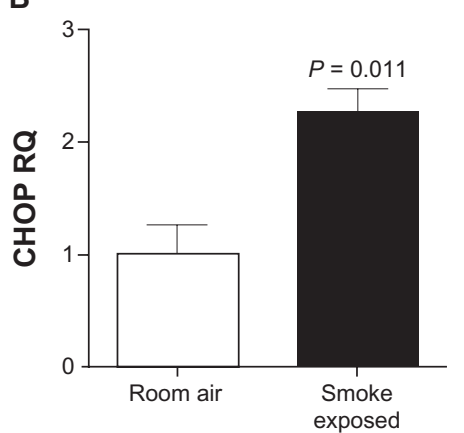

C

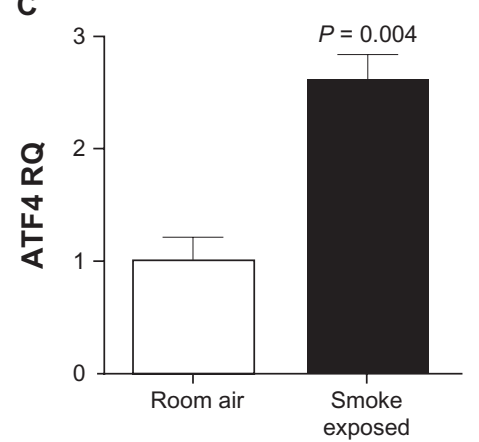

Figure 5 Mouse alveolar inflammatory cells are sensitive to cigarette smoke-induced ATF4/CHOP expression. C57BL/CBA mice exposed to cigarette smoke for 10 days were sacrificed and cells were collected from BAL fluid. A) Increased numbers of alveolar inflammatory were recovered from smoke exposed mice, compared to room air exposed littermates. qPCR was performed on cDNA generation from these cells and demonstrated increased levels of (B) CHOP and (C) ATF4 gene expression, corrected to beta actin levels.

Note: $P$-values shown, comparing mice exposed to room air (where $\mathrm{n}=4$ mice for each group).

Abbreviations: BAL, bronchoalveolar lavage; $\mathrm{CHOP}, \mathrm{C} / \mathrm{EBP}$ homologous protein. 
with inhibition of pre-adipocyte differentiation and a decrease in lipid accumulation. ${ }^{4}$ Whether the induction of ER stress following cigarette smoke exposure is sufficient to induce disease requires further investigation. The most striking evidence in the recent literature for cigarette smoke inducing ER stress is observed when examining severe COPD, with advanced stages of COPD having greater levels of ER stress, cell apoptosis, and loss of NF-E2-related factor 2 (Nrf2) expression. ${ }^{8}$ The study by Malhotra et al also demonstrated a trend for cigarette smoke-induced ER stress in wild-type mice, ${ }^{8}$ similar to our study. However, loss of Nrf2 expression in mice is required for cigarette smoke to significantly induce ER stress markers expression compared to wild-type mice. ${ }^{8}$ COPD patients have a decrease in Nrf2-dependent proteasomal subunits and total proteasomal activity and greater level of ER stress markers. ${ }^{8}$ The increase in the ER stress observed in GOLD III/IV stage COPD patients is associated with aberrant proteostasis ${ }^{7}$ and may be a means of causing the increased ER stress observed in the literature. ${ }^{7,8}$ Interestingly, overexpression of Nrf2-regulated proteasomal subunit PSMB6 inhibits cigarette smoke condensate induction of ER stress and subsequent Beas $2 \mathrm{~B}$ cell death. ${ }^{8}$ But the following question still remains, is this increase in ER stress induced solely by the cigarette smoke and does the process contribute to pathogenesis of the disease? Increased ER stress has been implicated to play a major role in other lung diseases, such as IPF, ${ }^{27,28}$ cystic fibrosis, ${ }^{29}$ and emphysema in individuals deficient for alpha one antitrypsin. ${ }^{30,31}$ We did observe a trend change in CHOP gene expression within the whole lung and a significant increase in inflammatory cells from BAL fluid. Malhotra and colleagues demonstrated that cigarette smoke-induced caspase activity appears to be independent of $\mathrm{CHOP}$ expression in wild-type mice ${ }^{8}$ however, it is still unclear whether cigarette smoke-induced $\mathrm{CHOP}$ expression has a pro-survival or pro-apoptotic effect.

The UPR is an important mechanism implemented in eukaryotic cells to restore ER homeostasis following an imbalance between the demand on the ER to load client proteins and its capacity to do so. ${ }^{32}$ Although ER stress and oxidative stress are closely linked events, the molecular pathways that couple these processes are poorly understood. It appears that the UPR is deliberately organized to minimize the stress of oxidative protein folding. ${ }^{26}$ Both reactive oxygen species (ROS) and unfolded protein are required to activate the UPR and subsequent apoptosis. ${ }^{33}$ Others have suggested that oxidative stress and ER stress are uncoupled, as oxidative stress could be effectively inhibited with antioxidants (alpha-tocopherol and ascorbic acid), but these antioxidants did not inhibit ER stress. ${ }^{34}$ Interestingly, cells with compromised ER function, such as defective UPR or ER-associated protein degradation, are susceptible to ROS production. ${ }^{26,35}$ In addition, ROS are an essential component in the events leading to protein misfolding in the ER and ER stress-induced apoptosis. Cigarette smokeinduced-ROS could exacerbate protein misfolding in the ER lumen by oxidizing amino acids in folding proteins or modifying chaperone and/or ER associated protein degradation (ERAD) functions, thereby amplifying UPR signaling.

Since COPD is an in vivo phenomenon, our approach to investigate the UPR in two animal models highlights the importance of a translational approach, utilizing in vitro investigations, animal models, and human samples to examine a multi-faceted disease that is the third leading cause of death in the USA. Similar to the cellular response to CSE, cigarette smoke exposure gives a trend increase in CHOP expression. Surprisingly, we do find reduced CHOP and ATF4 expression in mouse lungs following 12 months of smoke exposure. Perhaps an early induction of $\mathrm{CHOP}$ is required in disease progression and the expression of CHOP in inflammatory cells, such as macrophages, may contribute to disease progression. ER stress in macrophage has been predominately associated with disease progression in atherosclerosis ${ }^{36,37}$ and represents an intriguing area of investigation in cigarette smoke-induced diseases. This is highlighted by an induction of CHOP expression when examining isolate inflammatory cells compared to whole lung homogenates. Additionally, chronic cigarette smoke-induced ER stress observed in the human disease may be dependent on subsequent loss of other important factors, such as Nrf2 as impaired Nrf2 signaling causes a decline in proteasomal activity and increases the ER stress response in lungs of cigarette smoked mice and COPD patients. ${ }^{8}$

\section{Conclusion}

In conclusion, we have demonstrated that several populations of nontransformed human cells are moderately sensitive to cigarette smoke-induced ER stress. We do however observe a significant increase in ER stress in lung inflammatory cells from mice exposed to cigarette smoke. Additionally, it appears that the PERK/ATF4 branch is possibly the primary signaling pathway responsible for cigarette smoke-induced CHOP. The trend in CHOP induction may contribute to the pathogenesis of COPD but further examination of this concept 
is required. We have observed a slight in vivo induction of ER stress following acute exposure to cigarette smoke in guinea pig whole lung homogenate. However, in mice, chronic exposure to cigarette smoke reduces CHOP expression. Future studies are warranted to determine the effect of ER stress on COPD progression, with specific emphasis on cell specificity. The ultimate answer to the importance of ER stress in the development of disease needs to be addressed by examining the conditional knockouts of critical ER stress markers, such as CHOP or GRP78, in the presence of short and long-term smoke exposure. Additionally, treatment with an ER stress inhibitor, such as salubrinal ${ }^{7}$ or an antioxidant ${ }^{33}$ under cigarette smoking conditions in animal models, may provide means of assessing the possibility of targeting ER stress in COPD and provide a feasible means of treating this devastating disease.

\section{Acknowledgments}

This study was supported by NIHRO1 HL086936 (J D'Armiento). P Geraghty is supported by a T32 training grant from the NIH (HL007343). The authors are grateful to Robert Foronjy and Tracie DeVries-Seimon for useful discussions about study design.

\section{Disclosure}

The authors report no conflicts of interest in this work.

\section{References}

1. Pauwels RA, Buist AS, Ma P, Jenkins CR, Hurd SS, Committee GS. Global strategy for the diagnosis, management, and prevention of chronic obstructive pulmonary disease: National Heart, Lung, and Blood Institute and World Health Organization Global Initiative for Chronic Obstructive Lung Disease (GOLD): executive summary. Respir Care. 2001;46(8): 798-825.

2. Miniño AM, Xu J, Kochanek KD. Deaths: preliminary data for 2008. National Vital Statistics Reports. 2010;59(2):1-72.

3. Hogg JC, Chu F, Utokaparch S, et al. The nature of small-airway obstruction in chronic obstructive pulmonary disease. $N$ Engl J Med. 2004; 350(26):2645-2653.

4. Shimada T, Hiramatsu N, Hayakawa K, et al. Dual suppression of adipogenesis by cigarette smoke through activation of the aryl hydrocarbon receptor and induction of endoplasmic reticulum stress. Am J Physiol Endocrinol Metab. 2009;296(4):E721-E730.

5. Jorgensen E, Stinson A, Shan L, Yang J, Gietl D, Albino AP. Cigarette smoke induces endoplasmic reticulum stress and the unfolded protein response in normal and malignant human lung cells. BMC Cancer. 2008; 8:229.

6. Tagawa Y, Hiramatsu N, Kasai A, et al. Induction of apoptosis by cigarette smoke via ROS-dependent endoplasmic reticulum stress and CCAAT/enhancer-binding protein-homologous protein (CHOP). Free Radic Biol Med. 2008;45(1):50-59.

7. Min T, Bodas M, Mazur S, Vij N. Critical role of proteostasis-imbalance in pathogenesis of COPD and severe emphysema. $J$ Mol Med. $2011 \mathrm{Feb}$ 12. [Epub ahead of print].

8. Malhotra D, Thimmulappa R, Vij N, et al. Heightened endoplasmic reticulum stress in the lungs of patients with chronic obstructive pulmonary disease: the role of Nrf2-regulated proteasomal activity. Am J Respir Crit Care Med. 2009;180(12):1196-1207.
9. Kelsen SG, Duan X, Ji R, Perez O, Liu C, Merali S. Cigarette smoke induces an unfolded protein response in the human lung: a proteomic approach. Am J Respir Cell Mol Biol. 2008;38(5):541-550.

10. Hengstermann A, Muller T. Endoplasmic reticulum stress induced by aqueous extracts of cigarette smoke in $3 \mathrm{~T} 3$ cells activates the unfoldedprotein-response-dependent PERK pathway of cell survival. Free Radic Biol Med. 2008;44(6):1097-1107.

11. Dorner AJ, Wasley LC, Kaufman RJ. Protein dissociation from GRP78 and secretion are blocked by depletion of cellular ATP levels. Proc Natl Acad Sci U S A. 1990;87(19):7429-7432.

12. Harding HP, Calfon M, Urano F, Novoa I, Ron D. Transcriptional and translational control in the Mammalian unfolded protein response. Annu Rev Cell Dev Biol. 2002;18:575-599.

13. Marciniak SJ, Garcia-Bonilla L, Hu J, Harding HP, Ron D. Activationdependent substrate recruitment by the eukaryotic translation initiation factor 2 kinase PERK. J Cell Biol. 2006;172(2):201-209.

14. Kassenbrock CK, Garcia PD, Walter P, Kelly RB. Heavy-chain binding protein recognizes aberrant polypeptides translocated in vitro. Nature. 1988;333(6168):90-93.

15. Watterson TL, Hamilton B, Martin R, Coulombe RA Jr. Urban particulate matter causes ER stress and the unfolded protein response in human lung cells. Toxicol Sci. 2009;112(1):111-122.

16. Mercer BA, Kolesnikova N, Sonett J, D'Armiento J. Extracellular regulated kinase/mitogen activated protein kinase is up-regulated in pulmonary emphysema and mediates matrix metalloproteinase- 1 induction by cigarette smoke. J Biol Chem. 2004;279(17):17690-17696.

17. Foronjy RF, Mercer BA, Maxfield MW, Powell CA, D’Armiento J, Okada Y. Structural emphysema does not correlate with lung compliance: lessons from the mouse smoking model. Exp Lung Res. 2005; 31(6):547-562.

18. Foronjy RF, Mirochnitchenko O, Propokenko O, et al. Superoxide dismutase expression attenuates cigarette smoke- or elastase-generated emphysema in mice. Am J Respir Crit Care Med. 2006;173(6): 623-631.

19. Golovatch P, Mercer BA, Lemaitre V, Wallace A, Foronjy RF, D'Armiento J. Role for cathepsin $\mathrm{K}$ in emphysema in smoke-exposed guinea pigs. Exp Lung Res. 2009;35(8):631-645.

20. Yoshida H, Matsui T, Yamamoto A, Okada T, Mori K. XBP1 mRNA is induced by ATF6 and spliced by IRE1 in response to ER stress to produce a highly active transcription factor. Cell. 2001;107(7):881-891.

21. Beasley MB. Smoking-related small airway disease - a review and update. Adv Anat Pathol. 2010;17(4):270-276.

22. Bourdin A, Kotsimbos T, Nguyen K, et al. Non-invasive assessment of small airway remodelling in smokers. COPD. 2010;7(2):102-110.

23. Pauly JL, Smith LA, Rickert MH, Hutson A, Paszkiewicz GM. Review: Is lung inflammation associated with microbes and microbial toxins in cigarette tobacco smoke? Immunol Res. 2010;46(1-3):127-136.

24. Calfon M, Zeng H, Urano F, et al. IRE1 couples endoplasmic reticulum load to secretory capacity by processing the XBP-1 mRNA. Nature. 2002;415(6867):92-96.

25. MaY, Hendershot LM. Herp is dually regulated by both the endoplasmic reticulum stress-specific branch of the unfolded protein response and a branch that is shared with other cellular stress pathways. J Biol Chem. 2004;279(14):13792-13799.

26. Harding HP, Zhang Y, Zeng H, et al. An integrated stress response regulates amino acid metabolism and resistance to oxidative stress. Mol Cell. 2003;11(3):619-633.

27. Korfei M, Ruppert C, Mahavadi P, et al. Epithelial endoplasmic reticulum stress and apoptosis in sporadic idiopathic pulmonary fibrosis. $\mathrm{Am}$ J Respir Crit Care Med. 2008;178(8):838-846.

28. Lawson WE, Crossno PF, Polosukhin VV, et al. Endoplasmic reticulum stress in alveolar epithelial cells is prominent in IPF: association with altered surfactant protein processing and herpesvirus infection. Am J Physiol Lung Cell Mol Physiol. 2008;294(6):L1119-L1126.

29. Knorre A, Wagner M, Schaefer HE, Colledge WH, Pahl HL. DeltaF508-CFTR causes constitutive NF-kappaB activation through an ER-overload response in cystic fibrosis lungs. Biol Chem. 2002;383(2): 271-282. 
30. Lawless MW, Mankan AK, Gray SG, Norris S. Endoplasmic reticulum stress - a double edged sword for Z alpha-1 antitrypsin deficiency hepatoxicity. Int J Biochem Cell Biol. 2008;40(8):1403-1414.

31. Kelly E, Greene CM, Carroll TP, McElvaney NG, O’Neill SJ. Selenoprotein S/SEPS1 modifies endoplasmic reticulum stress in Z variant alpha1-antitrypsin deficiency. J Biol Chem. 2009;284(25): 16891-16897.

32. Shen X, Zhang K, Kaufman RJ. The unfolded protein response-a stress signaling pathway of the endoplasmic reticulum. J Chem Neuroanat. 2004;28(1-2):79-92.

33. Malhotra JD, Miao H, Zhang K, et al. Antioxidants reduce endoplasmic reticulum stress and improve protein secretion. Proc Natl Acad Sci US A. 2008;105(47):18525-18530.
34. Sheikh-Ali M, Sultan S, Alamir AR, Haas MJ, Mooradian AD. Effects of antioxidants on glucose-induced oxidative stress and endoplasmic reticulum stress in endothelial cells. Diabetes Res Clin Pract. 2010;87(2):161-166. [Epub 2009 Nov 24].

35. Haynes CM, Titus EA, Cooper AA. Degradation of misfolded proteins prevents ER-derived oxidative stress and cell death. Mol Cell. 2004;15(5):767-776.

36. Thorp E, Iwawaki T, Miura M, Tabas I. A reporter for tracking the unfolded protein response in vivo reveals patterns of temporal and cellular stress during atherosclerotic progression. J Lipid Res. 2011;52(5): 1033-1038. [Epub 2011 Feb 25].

37. Tabas I. The role of endoplasmic reticulum stress in the progression of atherosclerosis. Circ Res. 2010;107(7):839-850.

\section{Publish your work in this journal}

The International Journal of COPD is an international, peer-reviewed journal of therapeutics and pharmacology focusing on concise rapid reporting of clinical studies and reviews in COPD. Special focus is given to the pathophysiological processes underlying the disease, intervention programs, patient focused education, and self management protocols.

\section{Dovepress}

This journal is indexed on PubMed Central, MedLine and CAS. The manuscript management system is completely online and includes a very quick and fair peer-review system, which is all easy to use. Visit http://www.dovepress.com/testimonials.php to read real quotes from published authors.

Submit your manuscript here: http://www.dovepress.com/international-journal-of-copd-journal 